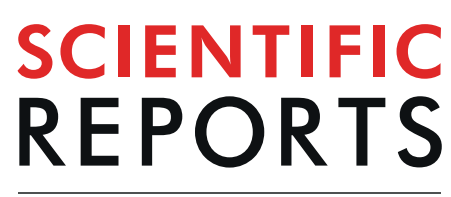

natureresearch

\title{
OPEN Therapeutic outcomes of non-grafted and platelet concentrations-grafted transcrestal maxillary sinus elevation (TSFE): a systematic review and meta- analysis
}

\begin{abstract}
Tianqi Guo ${ }^{1}$, Karan Gulati ${ }^{1}$, Ziyun Shen ${ }^{2,3}$, Pingping $\operatorname{Han}^{1}$ \& Zhen Fan ${ }^{2,3^{*}}$
To evaluate and compare the stability, quantity and quality of bone augmentation at maxillary sinus elevation sites by non-grafted transcrestal sinus floor elevation (TSFE) and platelet concentration grafted transcrestal sinus floor elevation (PC-TSFE). A complete literature search was performed up to April 2019. Clinical controlled trials, retrospective cohort studies, and prospective cohort studies were selected based on inclusion criteria. The clinical outcomes were implant survival rate (ISR), marginal/ crestal bone loss (MBL/CBL) and endo-sinus bone gain (ESBG). Meta-analysis was conducted on these 1-year based values. Furthermore, another meta-analysis on 1-year ISR value was conducted among studies with different residual bone heights $(\mathrm{RBH})$ within the non-grafted TSFE group. A total of 18 studies were included: 13 in TSFE group and 5 in PC-TSFE group. No significant differences were displayed between the 1-year ISR of TSFE $(97 \%, 95 \% \mathrm{Cl}=0.96-0.99)$ and PC-TSFE group $(99 \%$, $95 \% \mathrm{Cl}=0.97-1.00)$. Among the various studies with different $\mathrm{RBH}$ within TSFE group, no significant differences in 1-year ISR were displayed. The 1-year MBL/CBL value of PC-TSFE group $(0.73 \mathrm{~mm}$, $95 \% \mathrm{Cl}=0.43-1.13 \mathrm{~mm})$ did not show significant difference as compared to TSFE group $(0.60 \mathrm{~mm}$, $95 \% \mathrm{Cl}=0.10-1.10 \mathrm{~mm}$ ). Furthermore, no significant enhancement was observed on 1-year ESBG value on PC-TSFE group $(3.51 \mathrm{~mm}, 95 \% \mathrm{Cl}=2.31-4.71 \mathrm{~mm})$ in comparison with the TSFE group $(2.87 \mathrm{~mm}$, $95 \% \mathrm{Cl}=2.18 \mathrm{~m}-3.55 \mathrm{~mm})$. Grafting platelet concentrations around dental implants at TSFE sites did not significantly enhance the adjacent bone regeneration. Moreover, TSFE was shown to be a reliable therapeutic option for implant sites that need simultaneous maxillary sinus augmentation, even under limited RBH.
\end{abstract}

The maxillary sinus is a cavity of pyramid shape in the maxilla with a volume of $12-15 \mathrm{~mL}$. Its anterior border extends into the premolar roots or distal surface of canine roots, and the posterior border reaches the maxillary tuberosity $^{1,2}$. Due to its structure and location, the maxillary sinus sometimes challenges the proper placement of the implant and also compromises its long-term functioning ${ }^{2}$.

To address this challenge and establish an adequate bone site for implantation, direct (lateral approach) maxillary sinus lift has been suggested since its first introduction in the $1980 \mathrm{~s}^{3}$. That lateral approach is reliable to augment large quantity of bone at surgical sites, however it has strict limitations including excessive surgical trauma and prolonged healing time, which must be addressed ${ }^{3}$. In that attempt, transcrestal maxillary sinus elevation (TSFE), which is proceeded via alveolar crest, has been suggested and well implemented in clinical dentistry ${ }^{4}$. It compresses and apically pushes the maxillary bone from alveolar crest, and thus elevates maxillary sinus

\footnotetext{
${ }^{1}$ The University of Queensland, School of Dentistry, Herston, QLD 4006, Australia. ${ }^{2}$ Shanghai Engineering Research Center of Tooth Restoration and Regeneration, Shanghai, 200072, PR China. ${ }^{3}$ Department of Oral Implant, School and Hospital of Stomatology, Tongji University, Shanghai, 200072, PR China. *email: miss.fanzhen@tongji.edu.cn
} 
membrane for bone substitutes and implants ${ }^{4}$. The TSFE enhances the bone density around implant surfaces by compressing the alveolar bone, thereby providing the implant with enhanced primary stability ${ }^{5-8}$.

Although the TSFE is easy to handle with reduced surgical trauma compared with lateral maxillary sinus lift ${ }^{5,6}$, it has few limitations including inability to directly visualize membrane augmentation procedures and increased risk of membrane perforations on the TSFE sites. Furthermore, it is also unsuitable for cases with severely compromised bone quantity $(\mathrm{RBH}<4 \mathrm{~mm})^{5,6}$. $\mathrm{RBH}$ is one of the critical factors, together with bone quality/density, general health conditions, alignment of adjacent teeth and others, which determine the selection of implant surgery modality ${ }^{2}$. For cases with limited $\mathrm{RBH}(<8 \mathrm{~mm})$, applying short implant or tilted implantation are two plausible options, however the optimized treatment modality is still TSFE, which significantly increases the available bone quantity and thus guarantee a sufficient osseointegration region?.

Various clinical studies have reported that TSFE on cases with limited RBH (4-6 mm) leads to favorable implant survival rates, as long as these cases can attain secured primary stability ${ }^{7,8}$, however the lateral/direct access of MSFA still remains as a "gold standard" for such cases with severely compromised bone quantity. This is attributed to limited RBH resulting in unsecured primary stability around the implants, and the inability of TSFE to provide direct visualization of membrane augmentation, thus increasing the risk of membrane penetration during surgery ${ }^{6,10}$. As is indicated by previous studies, grafting bone substitutions is not essential for a successful TSFE, mainly because implants and surrounding bone usually protrude into the maxillary sinus, which in turn hold the Schneiderian membrane and maintains a stable chamber for osteogenesis ${ }^{11}$. Furthermore, bone grafts and sharp tip of the implants inserted into maxillary sinus may penetrate the Schneiderian membrane and result in compromised bone healing ${ }^{4,11}$. However, research gaps remain unaddressed including lack of investigations to find an appropriate osteoinductive bone substitutions, which is both soft and flexible (acts like buffer to protect the fragile Schneiderian membrane) into maxillary sinus in TSFE sites.

Platelet concentrations are autologous plasma portion that are extracted from the autologous whole blood and enriched with cytokines to promote tissue regeneration ${ }^{12-15}$. Previous studies have indicated that platelet concentrations include platelet-rich plasma (PRP), plate-rich fibrin (PRF), platelet-rich growth factor (PRGF) and concentrated growth factor (CGF), etc. ${ }^{12-15}$. Currently, these varied platelet concentrations are clinically applied to promote wound healing, accelerate bone regeneration and modulate the post-surgical inflammation ${ }^{16,17}$. Although there are some deviations among these products, such as components and amount of cytokines, these concentrations are mechanically flexible (either in liquid or gel-like phase) and able to promote wound healing at surgical sites ${ }^{12,13}$. Moreover, the interlocking mesh-like microstructure created by the aggregation of platelets make the platelet grafts effective in promoting the migration and proliferation of osteoprogenitor cells and thus can enhance the osseointegration at the implant-bone interface ${ }^{14}$. Based on the fact that platelet concentrations are soft and contain numerous cytokines to promote bone regeneration, they have been reported as grafting materials into maxillary sinus lift sites by many studies ${ }^{7,18-21}$.

The aim of this study is to explore the therapeutic outcomes between non-grafted and platelet concentrations grafted TSFE. The vertical quantity and stability of newly regenerated bone in both grafted and non-grafted groups were evaluated by analyzing their implant survival rate, marginal bone loss (MBL) and endo-sinus bone gain (ESBG). Meanwhile, to evaluate the clinical reliability of TSFE under limited RBH, the implant survival rates among subgroups of different average RBH within TSFE group were also investigated. In summary, this study will provide insight into the clinical application of platelet concentrations as grafting materials in TSFE sites, and the therapeutic reliability of non-grafted TSFE in sites with poor bone quantity.

\section{Results}

Literature search and selection. The process for searching and selecting literature is shown in Fig. 1. A total of 1891 studies were searched and saved in Endnote X9 $(n=1891)$. After 978 duplications were removed, title of residual literatures $(n=913)$ were scanned and identified, which led to removal of 746 , which did not fulfil the inclusion criteria. Then abstract assessments were performed on the remaining studies $(n=167)$, from which 136 studies were excluded. A final full-text reading was conducted on the remaining studies $(n=31)$. Furthermore, a total of 13 articles were excluded for the following reasons: 5 for repeat with subsequent studies $^{22-26}, 3$ for inconsistent follow-up visit time ${ }^{27-29}, 3$ for the inaccurate and insufficient data ${ }^{30-32}, 1$ where grafted material did not fulfil the inclusion criteria ${ }^{33}$, and 1 was excluded for the inaccurate demographic data ${ }^{34}$. Finally, 18 studies were included in this systematic review and meta-analysis, among which were 13 non-grafted TSFE ${ }^{35-}$ 47, 5 TSFE grafted with various platelet concentrations (PRP, PRF, CGF or PRGF) ${ }^{7,18-21}$.

Literature quality assessments. Tables 1 and 2 shows the literature quality assessments of the 3 controlled studies and 15 non-randomized studies. For the controlled studies, the literature reported by Si et al. in 2013 qualified as low risk of bias, while the studies by Lai H C (2010) and Nedir (2017) were regarded with moderate risk of bias. For the non-randomized studies (prospective and retrospective cohort studies), all the 15 studies scored 7 or more and were assessed as high quality with low risk of bias.

Demographics and surgical methods. As is presented in Table 3, among the 13 studies on non-grafted TSFE, Straumann implants were applied in 6 studies; Astra-tech implants were applied in 3 studies; 2 used both Neoss and Nobel Biocare implant systems; and 1 study used both Osstem and Bego implant systems. Furthermore, 1 study by Lai et al. did not clearly describe the implants' brand name. There were 6 retrospective studies, 4 prospective studies, 2 randomized controlled tests and 1 clinical controlled test for the non-grafted TSFE group. All the 3 controlled tests compared the therapeutic outcomes of non-grafted TSFE with TSFE grafted with bone substitutions, and for these studies the data of the non-grafted group were extracted and analyzed in the meta-analysis. 5 studies had reported the platelet-grafted TSFE (Table 3), among which were 2 retrospective studies, 2 prospective studies, while the other 1 was not clearly defined. Considering the various grafted platelet 


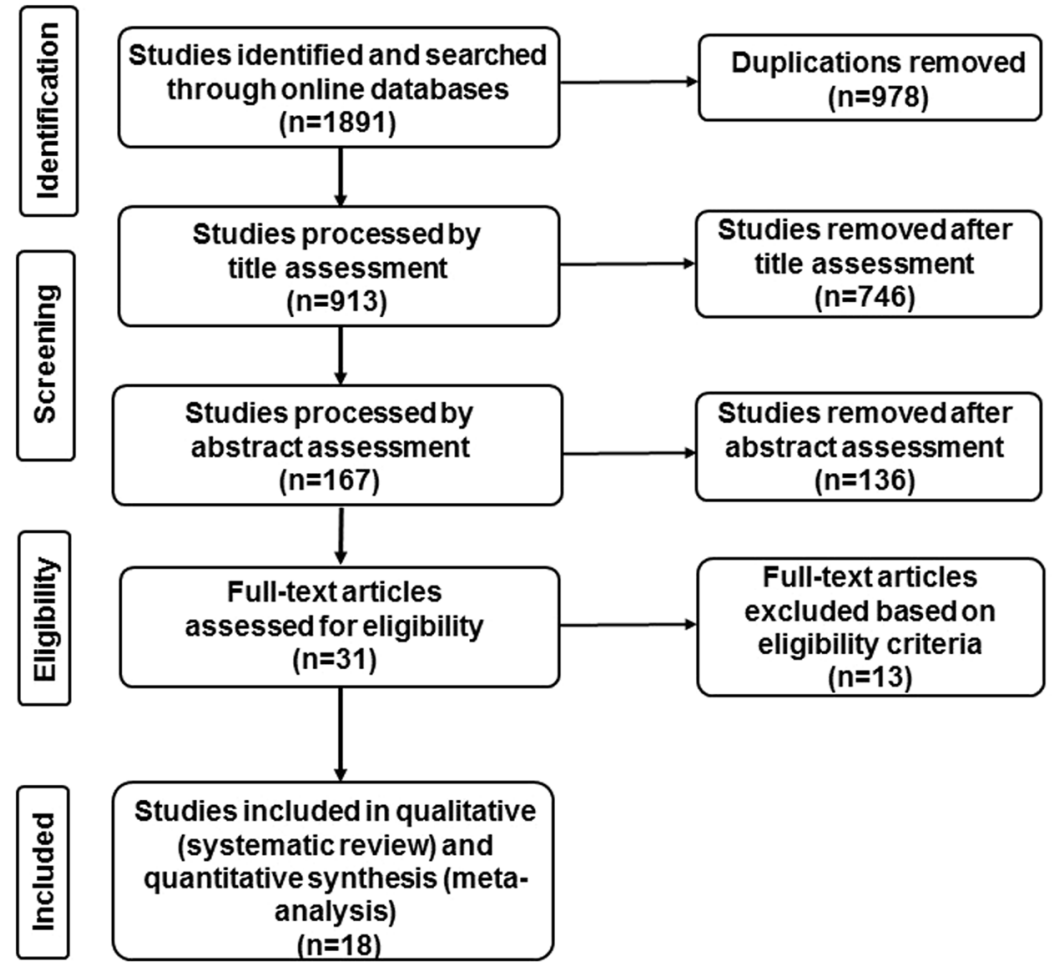

Figure 1. Flow diagram depicting the literature identification and selection in the current study.

\begin{tabular}{|l|l|l|l|l|l|l|l|l|}
\hline Author (Year) & $\begin{array}{l}\text { Adequate } \\
\text { sequence } \\
\text { generation }\end{array}$ & $\begin{array}{l}\text { Allocation } \\
\text { concealment }\end{array}$ & $\begin{array}{l}\text { Blinding of } \\
\text { participants } \\
\text { and personnel }\end{array}$ & $\begin{array}{l}\text { Blinding } \\
\text { of outcome } \\
\text { assessment }\end{array}$ & $\begin{array}{l}\text { Incomplete } \\
\text { outcome data } \\
\text { addressed }\end{array}$ & $\begin{array}{l}\text { Selective } \\
\text { outcome } \\
\text { reporting }\end{array}$ & $\begin{array}{l}\text { Free from } \\
\text { other bias }\end{array}$ \\
\hline Si M (2013) & Yes & Yes & Yes & Yes & Yes & Yes & Yes & Low \\
\hline Lai H (2010) & No & Yes & No & No & Yes & Yes & Yes & Moderate \\
\hline Nedir (2017) & Yes & Yes & No & No & Yes & Yes & Yes & Moderate \\
\hline
\end{tabular}

Table 1. The literature quality and risk of bias assessment of controlled studies by Cochrane scale form.

concentrations, 2 of them were PRGF, the other 3 were PRP, PRF and CGF, respectively. 2 studies applied BTI implant system, 2 studies applied Zimmer and Astra-tech implants, respectively, and the other 1 was not clearly indicated.

Implant survival rate. As presented in Table 3, for the non-grafted TSFE group, the cumulated implant survival rates ranged between $90.6 \%$ and $100 \%$, within the varied follow-up visit time (6-120 months). For the studies with longest follow-up visit time (10 years), the 10 -year cumulative implant survival rate was $100 \%$ in Nedir's study (25 implants), and 91.2\% in Caban's study (31/34 implants, 3 failed implants occurred within the $1^{\text {st }}$ year) ${ }^{35,36}$. The lowest implant survival rate was $90.6 \%$ (87 out of 96 implants survived) over 108 months, as is reported by $\mathrm{Si}$ et al. ${ }^{38}$. For the platelet-grafted TSFE, 4 out of 5 studies reported their 1 -year implant survival rates, which ranged between $97.1 \%$ and $100 \%^{7,18-20}$. The only failed implant occurred in the study by Diss A et al., which reported 1 out of 35 implants failed at 1 year after treatment with PRF-grafted TSFE ${ }^{21}$. The meta-analysis on the 1 -year implant-based survival rate of non-grafted TSFE showed an average value of $97 \%(95 \% \mathrm{CI}=0.96-0.99)$, while the PC-TSFE was $99 \%(95 \% \mathrm{CI}=0.97-1.00)$ (Fig. 2). Although slightly higher average ISR was obtained in the PC-TSFE group, the non-grafted TSFE group still obtained a favorable 1-year ISR, which indicated that it is a reliable treatment modality for augmenting bone quantity in maxillary edentulous region. Furthermore, another meta-analysis on 1-year implant-based survival rate among different RBH within non-grafted TSFE studies indicated that the survival rate for studies with $\mathrm{RBH}<4 \mathrm{~mm}$ was $97 \%$ (95\%CI $0.92-1.00$ ), for whose RBH $=4 \sim 6 \mathrm{~mm}$ was $98 \%$ (95\%CI $0.94-1.00)$, and those with RBH $>6 \mathrm{~mm}$ was $97 \%$ (95\%CI 0.95-0.99) (Fig. 3). Finally, all the subgroups obtained favorable 1-year ISR values, which indicated that insufficient RBH is not restriction for non-grafted TSFE on maxillary edentulous region.

Marginal bone loss. 1-year marginal bone loss (MBL) has been reported by 7 studies in non-grafted TSFE, with the majority of them (5 out of 7) being less than $1 \mathrm{~mm}$. Spinelli et al. and Fermergrad et al. reported the smallest 1-year MBL, which were $0.33 \pm 0.36 \mathrm{~mm}$ and $0.5 \pm 0.06 \mathrm{~mm}$, respectively ${ }^{26,40}$. While the largest 1 -year MBL occurred in the study by Si et al. $(1.28 \pm 0.05 \mathrm{~mm})$ and Nedir et al. $(1.2 \pm 0.7 \mathrm{~mm})^{36,38}$. As presented in 


\begin{tabular}{|c|c|c|c|c|c|c|c|c|c|}
\hline \multirow[b]{2}{*}{ Author (Year) } & \multicolumn{4}{|l|}{ Selection } & \multirow{2}{*}{$\begin{array}{l}\text { Comparability } \\
\text { Comparability on } \\
\text { design or analysis }\end{array}$} & \multicolumn{3}{|l|}{ Outcome } & \multirow[b]{2}{*}{ Total } \\
\hline & $\begin{array}{l}\text { A representative of } \\
\text { the exposed cohort }\end{array}$ & $\begin{array}{l}\text { Selection } \\
\text { of external } \\
\text { control }\end{array}$ & $\begin{array}{l}\text { Ascertainment of } \\
\text { exposure }\end{array}$ & $\begin{array}{l}\text { Outcomes not } \\
\text { present at the } \\
\text { start }\end{array}$ & & $\begin{array}{l}\text { Outcome } \\
\text { assessment }\end{array}$ & $\begin{array}{l}\text { Enough } \\
\text { follow-up } \\
\text { visit time }\end{array}$ & $\begin{array}{l}\text { Adequacy of } \\
\text { follow-up of } \\
\text { cohorts }\end{array}$ & \\
\hline Diss A (2008) & $\star$ & 0 & $\star$ & $\star$ & $\star 0$ & $\star$ & $\star$ & $\star$ & $7 / 9$ \\
\hline Schleier P (2008) & $\star$ & 0 & $\star$ & $\star$ & $\star 0$ & $\star$ & $\star$ & $\star$ & $7 / 9$ \\
\hline Siovio T (2011) & $\star$ & 0 & $\star$ & $\star$ & $\star \star$ & $\star$ & $\star$ & $\star$ & $8 / 9$ \\
\hline Fermergard (2012) & $\star$ & 0 & $\star$ & $\star$ & $\star 0$ & $\star$ & $\star$ & $\star$ & $8 / 9$ \\
\hline $\mathrm{He} \mathrm{L}(2012)$ & $\star$ & 0 & $\star$ & $\star$ & $\star \star$ & $\star$ & 0 & $\star$ & $7 / 9$ \\
\hline Vople S (2013) & $\star$ & 0 & $\star$ & $\star$ & $\star 0$ & $\star$ & $\star$ & $\star$ & $7 / 9$ \\
\hline Brizeula A (2014) & $\star$ & 0 & $\star$ & $\star$ & $\star \star$ & $\star$ & $\star$ & $\star$ & $8 / 9$ \\
\hline Kim J M (2014) & $\star$ & 0 & $\star$ & $\star$ & $\star 0$ & $\star$ & $\star$ & $\star$ & $7 / 9$ \\
\hline Stiovio T (2014) & $\star$ & 0 & $\star$ & $\star$ & $\star 0$ & $\star$ & $\star$ & $\star$ & $7 / 9$ \\
\hline Anitua.E (2015) & $\star$ & 0 & $\star$ & $\star$ & $\star \star$ & 0 & $\star$ & $\star$ & $7 / 9$ \\
\hline Spinelli.D (2015) & $\star$ & 0 & $\star$ & $\star$ & $\star \star$ & $\star$ & $\star$ & $\star$ & $8 / 9$ \\
\hline Gu Y X (2016) & $\star$ & 0 & $\star$ & $\star$ & $\star 0$ & $\star$ & $\star$ & $\star$ & $7 / 9$ \\
\hline Nedir R (2016) & $\star$ & 0 & $\star$ & $\star$ & $\star \star$ & $\star$ & $\star$ & $\star$ & $8 / 9$ \\
\hline Si M S (2016) & $\star$ & 0 & $\star$ & $\star$ & $\star 0$ & $\star$ & $\star$ & $\star$ & $7 / 9$ \\
\hline Caban J (2017) & $\star$ & 0 & $\star$ & $\star$ & $\star 0$ & $\star$ & $\star$ & $\star$ & $7 / 9$ \\
\hline
\end{tabular}

Table 2. The literature quality and risk of bias assessment of non-randomized studies by Newcastle-Ottawa scale form.

Table 3, the majority of MBL value remains stable 1-year post-surgery, except the study reported by Gu et al. ${ }^{39}$. For the 5 studies which reported long-term MBL ( $\geq 5$ years), the majority of them reported an average 5-year MBL that was less than $1 \mathrm{~mm}$ except for Gu's study $(1.54 \pm 1.00 \mathrm{~mm}) .2$ studies in the PC-TSFE group had reported the average 1-year MBL: (1) Siovio's et al. reported an average 1-year MBL of $0.35 \pm 0.25 \mathrm{~mm}$, and (2) Anitua et al. reported an average 1-year MBL of $0.86 \pm 0.49 \mathrm{~mm}^{7,18,19}$. Meta-analysis on 1-year MBL indicated that the group of non-grafted TSFE was $0.73 \mathrm{~mm}(95 \% \mathrm{CI} 0.43 \mathrm{~mm}-1.13 \mathrm{~mm})$, while for the PC-TSFE was $0.60 \mathrm{~mm}(95 \% \mathrm{CI}$ $0.10 \mathrm{~mm}-1.10 \mathrm{~mm}$ ) (Fig. 4). Calculations from the equation revealed changes of 1-year MBL value between non-grafted TSFE and PC-TSFE to be $0.13 \pm 3.36 \mathrm{~mm}$, and since 0 was contained in this $95 \%$ CI $(-0.21 \mathrm{~mm}$, $0.47 \mathrm{~mm}$ ), hence no significant differences were obtained between the 1-year MBL of PC-TSFE and non-grafted TSFE groups.

Endo-sinus bone gain (ESBG). 8 out of 12 studies on non-grafted TSFE reported ESBG after surgery. The lowest ESBG occurred in Aritza's study, which was $1.8 \pm 0.3 \mathrm{~mm}$ over 24 months, while the highest value was reported by Spinell, which was $6.4 \pm 1.6 \mathrm{~mm}$ over $36 \mathrm{months}^{40,41} .4$ studies reported the change of ESBG value during a sequential follow-up visit time, which showed to be stable 1-year post-surgery, except Si's study which showed a significantly enhanced ESBG at 3 year as compared to of 1 year ${ }^{42}$. In the PC-TSFE group, all 5 studies reported the postsurgical ESBG, and the highest value was $8.23 \pm 2.88 \mathrm{~mm}$ by Kim et al. at 14 -months post-surgery ${ }^{20}$. The meta-analysis on 1-year ESBG includes 3 studies, which excluded the study by Siovio and Kim et al. ${ }^{40-42}$. The results confirm that average 1-year ESBG was $2.87 \mathrm{~mm}$ for non-grafted TSFE (95\%CI $2.18 \mathrm{~m}$ $3.55 \mathrm{~mm}$ ), and $3.51 \mathrm{~mm}$ for the PC-TSFE (95\%CI 2.31-4.71 mm) (Fig. 5). As per the equation, changes of 1-year ESBG between non-grafted TSFE and PC-TSFE was $0.64 \pm 7.25 \mathrm{~mm}$, and since 0 was contained within its $95 \%$ CI $(-0.15 \mathrm{~mm}, 1.43 \mathrm{~mm})$, no significant enhancements were observedon ESBG value after the intervention (applying PC in TSFE sites).

\section{Discussion}

The chamber of augmented maxillary sinus created by the apical displacement of maxillary sinus membrane (Schneiderian membrane) has active osteogenic potential ${ }^{48,49}$. As previous study indicated, cells extracted from the Schneiderian membrane can express osteogenic differentiation cytokines including CD105, CD73 and $\mathrm{CD}_{166^{48}}$. Moreover, there also are numerous ALP and STRO-I positive staining cells that can be separated from the Schneiderian membrane, which revealed the existence of osteoprogenitor cells and osteoblasts ${ }^{49}$. Based on these facts, it is indicated that bone grafts are not mandatory for a successful MSFA, as long as we could maintain the integrity of Schneiderian membrane and obtain a stabilized chamber for bone regeneration ${ }^{50}$. Studies focused on non-grafted TSFE have been performed and confirmed that such technique is reliable for MSFA sites, even in cases with limited residual bone height $(\mathrm{RBH})^{51}$. Considering the longevity of non-grafted TSFE, some studies have even shown that there were no differences in implant survival rates between bone substitutions grafted and non-grafted TSFE sites ${ }^{8,11}$. However, for the lateral MSFA, bone grafts are still recommended as a beneficial strategy, for both improving the implant survival rates and maintaining the stability of ESBG ${ }^{52}$.

Platelet concentrations (PCs) are extracted by centrifuging autologous blood and formed by the co-aggregate process of platelets, and they could be fabricated into the liquid form including PRP, PRGF or gel-like substrates such as PRF and $\mathrm{CGF}^{9,53-55}$. All these concentrations are rich in various cytokines that promote tissue regeneration, which include vascular endothelial growth factor (VEGF), insulin growth factor (IGF) and platelet-derived growth factor (PDGF) $)^{12-15,56-59}$. The release of cytokines from platelet concentrations is effective towards 


\begin{tabular}{|c|c|c|c|c|c|c|c|c|c|c|}
\hline \multicolumn{11}{|c|}{ Non-Grafted TSFE } \\
\hline Author (year) & $\begin{array}{l}\text { Implant } \\
\text { brand }\end{array}$ & $\begin{array}{l}\text { Implant } \\
\text { numbers }\end{array}$ & $\begin{array}{l}\text { Patient } \\
\text { numbers }\end{array}$ & $\begin{array}{l}\text { Surgical } \\
\text { methods }\end{array}$ & RBH & Follow-up time & $\begin{array}{l}\text { Implant } \\
\text { survival rate }\end{array}$ & MBL/CBL & ESBG & $\begin{array}{l}\text { Study } \\
\text { category }\end{array}$ \\
\hline \multirow{3}{*}{ Caban J(2017) } & \multirow{3}{*}{ Astra Tech } & \multirow{3}{*}{34} & \multirow{3}{*}{25} & \multirow{3}{*}{ TSFE } & \multirow{3}{*}{$4.3 \pm 1.0 \mathrm{~mm}$} & 12 & $91.2 \%$ & $0.4 \pm 0.42$ & \multirow{3}{*}{ N/A } & \multirow{3}{*}{ Retrospective } \\
\hline & & & & & & 60 & $91.2 \%$ & $0.5 \pm 0.45$ & & \\
\hline & & & & & & 120 & $91.2 \%$ & $0.6 \pm 0.82$ & & \\
\hline \multirow{3}{*}{$\begin{array}{l}\text { Nedir } \\
(2017)\end{array}$} & \multirow{3}{*}{$\begin{array}{l}\text { Straumann } \\
\text { AG }\end{array}$} & \multirow{3}{*}{17} & \multirow{3}{*}{9} & \multirow{3}{*}{ TSFE } & \multirow{3}{*}{$2.4 \pm 0.9$} & 12 & $100 \%$ & $0.6 \pm 0.8$ & $3.9 \pm 1.0$ & \multirow{3}{*}{$\begin{array}{l}\text { Randomized } \\
\text { controlled } \\
\text { study }\end{array}$} \\
\hline & & & & & & 36 & $94.12 \%$ & $0.6 \pm 1.1$ & $4.1 \pm 1.0$ & \\
\hline & & & & & & 60 & $94.12 \%$ & $0.6 \pm 0.9$ & $3.8 \pm 1.0$ & \\
\hline \multirow{4}{*}{$\begin{array}{l}\text { Nedir } \\
(2016)\end{array}$} & \multirow{4}{*}{$\begin{array}{l}\text { Straumann } \\
\text { AG }\end{array}$} & \multirow{4}{*}{25} & \multirow{4}{*}{17} & \multirow{4}{*}{ TSFE } & \multirow{4}{*}{$5.4 \pm 2.3$} & 12 & $100 \%$ & $1.2 \pm 0.7$ & $2.5 \pm 1.2$ & \\
\hline & & & & & & 36 & $100 \%$ & $0.9 \pm 0.8$ & $3.1 \pm 1.5$ & Prosnective \\
\hline & & & & & & 60 & $100 \%$ & $0.8 \pm 0.8$ & $3.2 \pm 1.3$ & Prospective \\
\hline & & & & & & 120 & $100 \%$ & $1.0 \pm 0.9$ & $3.0 \pm 1.4$ & \\
\hline & & & & & & 12 & $96.2 \%$ & & & \\
\hline & & & & & & 24 & $94.8 \%$ & $\mathrm{~N} / \mathrm{A}$ & N/A & \\
\hline & & & & & & 36 & $92.7 \%$ & & & \\
\hline & & & & & & 48 & $92.7 \%$ & $0.46 \pm 0.88$ & $2.95 \pm 1.25$ & \\
\hline & & & & & $6.75 \pm 1.91 \mathrm{~mm}$ & 60 & $90.6 \%$ & $0.50 \pm 0.96$ & $3.01 \pm 1.36$ & Retrospective \\
\hline & & & & & & 72 & $90.6 \%$ & $0.50 \pm 0.97$ & $3.74 \pm 1.34$ & \\
\hline Si M S (2016) & Straumann & 96 & 80 & TSFE & & 84 & $90.6 \%$ & $0.46 \pm 1.08$ & $2.63 \pm 1.36$ & \\
\hline & & & & & & 96 & $90.6 \%$ & $0.48 \pm 1.32$ & $2.55 \pm 1.11$ & \\
\hline & & & & & & 108 & $90.6 \%$ & $0.50 \pm 1.69$ & $2.16 \pm 1.13$ & \\
\hline & & & & & $>=5 \mathrm{~mm}$ & 108 & $\begin{array}{l}93.5 \% \\
(\mathrm{n}=72)\end{array}$ & N/A & $2.89 \pm 1.16$ & Retrosnective \\
\hline & & & & & $<5 \mathrm{~mm}$ & 108 & $\begin{array}{l}78.9 \% \\
(n=15) \\
\end{array}$ & N/A & $3.24 \pm 1.63$ & Ketrospective \\
\hline & & & & & & 12 & $94.6 \%$ & $0.83 \pm 0.50$ & & \\
\hline Gu Y X (2016) & Straumann & 37 & 25 & TSFE & $2.81 \pm 0.74$ & 36 & $94.6 \%$ & $1.47 \pm 1.02$ & N/A & Prospective \\
\hline & & & & & & 60 & $94.6 \%$ & $1.54 \pm 1.00$ & & \\
\hline D.Spinelli & NobelSpeedy & 66 & 39 & Template & $67+16$ & 12 & $98.83 \%$ & $0.33 \pm 0.36$ & $\mathrm{~N} / \mathrm{A}$ & Prosnective \\
\hline$(2015)$ & NobelActive & 66 & 39 & guided TSFE & $0.7 \pm 1.0$ & 36 & $98.83 \%$ & $0.51 \pm 0.29$ & $6.4 \pm 1.6$ & Prospective \\
\hline $\begin{array}{l}\text { Aritza B } \\
(2014)\end{array}$ & $\begin{array}{l}\text { Straumann } \\
\text { Klockner }\end{array}$ & 36 & N/A & TSFE & $7.4 \pm 0.4$ & 24 & $91.6 \%$ & $0.7 \pm 0.1$ & $1.8 \pm 0.3$ & Prospective \\
\hline $\mathrm{SiMS}(2013)$ & Straumann & & 20 & TSFF & $458+147$ & 12 & $100 \%$ & $1.28 \pm 0.05$ & $2.56 \pm 0.98$ & Randomized \\
\hline Si M S(2013) & SLA & 20 & 20 & TSFE & $4.58 \pm 1.47$ & 36 & $95.0 \%$ & $1.38 \pm 0.23$ & $3.07 \pm 1.68$ & control test \\
\hline $\begin{array}{l}\text { Stefano V } \\
(2013)\end{array}$ & $\begin{array}{l}\text { Neoss Ltd } \\
\text { Harrogate }\end{array}$ & 29 & 20 & TSFE & $7.2 \pm 1.5$ & $11-32$ & $100 \%$ & $0.7 \pm 0.3$ & $2.8 \pm 1.2$ & Retrospective \\
\hline $\begin{array}{l}\text { He L } \\
(2013)\end{array}$ & $\begin{array}{l}\text { Bego } \\
\text { Osstem }\end{array}$ & 27 & 22 & TSFE & $6.7 \pm 1.2$ & 6 & $100 \%$ & $\mathrm{~N} / \mathrm{A}$ & $2.5 \pm 1.5$ & Retrospective \\
\hline Robert F & Astra & 53 & 36 & TSFF & & 12 & $96 \%$ & $0.5 \pm 0.06$ & N/A & Retresnective \\
\hline$(2012)$ & Astra & 53 & 36 & TSFE & $6.3 \pm 0.3$ & 36 & $94 \%$ & $0.6 \pm 0.09$ & N/A & Retrospective \\
\hline $\begin{array}{l}\text { Lai H C } \\
(2010)\end{array}$ & N/A & 191 & 125 & TSFE & $5.6 \pm 2.5$ & 60 & $97.38 \%$ & N/A & N/A & $\begin{array}{l}\text { Randomized } \\
\text { control test }\end{array}$ \\
\hline Peter S (2008) & \begin{tabular}{|l} 
Straumann SP \\
Straumann TE
\end{tabular} & 62 & 30 & $\begin{array}{l}\text { Endocsope } \\
\text { guided TSFE }\end{array}$ & $8.4 \pm 2.2$ & 24 & $94 \%$ & $\mathrm{~N} / \mathrm{A}$ & $3.5 \pm 1.8$ & Retrospective \\
\hline Platelet Conceı & ntrations Grafte & TSFE & & & & & & & & \\
\hline Author (year) & $\begin{array}{l}\text { Implant } \\
\text { brand }\end{array}$ & $\begin{array}{l}\text { Implant } \\
\text { numbers }\end{array}$ & $\begin{array}{l}\text { Patient } \\
\text { numbers }\end{array}$ & $\begin{array}{l}\text { Surgical } \\
\text { methods }\end{array}$ & $\begin{array}{l}\text { Residual bone } \\
\text { height }\end{array}$ & Follow-up time & $\begin{array}{l}\text { Implant } \\
\text { survival rate }\end{array}$ & $\begin{array}{l}\text { Marginal } \\
\text { bone loss }\end{array}$ & ESBG & $\begin{array}{l}\text { Study } \\
\text { category }\end{array}$ \\
\hline $\begin{array}{l}\text { E. Anitua } \\
(2015)\end{array}$ & $\mathrm{N} / \mathrm{A}$ & 61 & 48 & TSFE + PRGF & $4.03 \pm 0.51$ & 12 & $98.3 \%$ & $\begin{array}{l}0.86 \pm 0.49 \\
(\mathrm{n}=30)\end{array}$ & $4.64 \pm 1.68$ & Retrospective \\
\hline $\begin{array}{l}\text { Siovio T } \\
(2014)\end{array}$ & BTI & 65 & 25 & TSFE + PRP & $5.8+1.10$ & 12 & $100 \%$ & $0.35 \pm 0.25$ & $2.7 \pm 1.29$ & Prospective \\
\hline $\begin{array}{l}\text { Ji-Min Kim } \\
(2014)\end{array}$ & Zimmer & 16 & 11 & CGF + HPISE & $4.98 \pm 2.8$ & 14 & $100 \%$ & $\mathrm{~N} / \mathrm{A}$ & $8.23 \pm 2.88$ & Retrospective \\
\hline $\begin{array}{l}\text { Siovio } \\
(2011)\end{array}$ & BTI & 15 & 15 & TSFE + PRGF & N/A & $\begin{array}{l}24-50 \\
\text { (average 35.6) }\end{array}$ & $100 \%$ & $0.36 \pm 0.19$ & $2.9 \pm 0,8$ & $\mathrm{~N} / \mathrm{A}$ \\
\hline Diss A (2008) & Astra tech & 35 & 20 & TSFE + PRF & $6.5 \pm 1.7$ & 12 & $97.1 \%$ & N/A & $3.2 \pm 1.5$ & Prospective \\
\hline
\end{tabular}

Table 3. Demographic data and therapeutic outcomes of studies included in the final systematic review and meta-analysis. TSFE: transcrestal sinus floor elevation; RBH: residual bone height; $\mathrm{MBL} / \mathrm{CBL}$ : marginal/crestal bone loss; ESBG: endo-sinus bone gain. 


\begin{tabular}{|c|c|c|c|}
\hline Study & & & $\%$ \\
\hline ID & & $\mathrm{ES}(95 \% \mathrm{CI})$ & Weight \\
\hline Non-Grafted & $\mathrm{i}$ & & \\
\hline Caban J (2017) & $\rightarrow 1$ & $0.90(0.80,1.00)$ & 1.07 \\
\hline Nedir (2017) & $\rightarrow$ & $0.99(0.93,1.04)$ & 3.46 \\
\hline Nedir (2016) & $\leftarrow$ & $0.99(0.95,1.03)$ & 7.35 \\
\hline Si M S (2016) & $\leftarrow$ & $0.96(0.93,1.00)$ & 7.84 \\
\hline Gu Y X (2016) & $\rightarrow+1$ & $0.93(0.86,1.01)$ & 1.72 \\
\hline D.Spinelli (2015) & $\leftarrow$ & $0.98(0.94,1.01)$ & 8.76 \\
\hline Si M S (2013) & $\rightarrow$ & $0.99(0.94,1.04)$ & 4.75 \\
\hline Robert F (2012) & $\rightarrow$ & $0.95(0.90,1.01)$ & 3.42 \\
\hline Subtotal $(\mathrm{I}-\mathrm{squared}=0.0 \%, \mathrm{p}=0.663)$ & $\phi$ & $0.97(0.96,0.99)$ & 38.37 \\
\hline - & 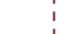 & & \\
\hline PC Grafted & 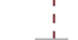 & & \\
\hline E.Anitua (2015) & $\rightarrow$ & $0.98(0.94,1.01)$ & 7.51 \\
\hline Siovio T (2014) & - & $1.00(0.98,1.01)$ & 48.50 \\
\hline Ji Min Kim (2014) & $\vec{\imath}$ & $0.99(0.93,1.04)$ & 3.08 \\
\hline Diss A (2008) & $\rightarrow$ & $0.96(0.89,1.02)$ & 2.54 \\
\hline Subtotal $(\mathrm{I}-\mathrm{squared}=0.0 \%, \mathrm{p}=0.584)$ & 0 & $0.99(0.98,1.00)$ & 61.63 \\
\hline . & i & & \\
\hline Overall $(I-$ squared $=0.0 \%, p=0.552)$ & f & $0.98(0.97,1.00)$ & 100.00 \\
\hline NOTE: Weights are from random effects analysis & i & & \\
\hline-1.04 & 1.0 & & \\
\hline
\end{tabular}

Figure 2. The meta-analysis on 1-year implant survival rate for the non-grafted TSFE and the PC-TSFE. Forest plot was generated by Stata 14.0, and randomized model was applied for the meta-analysis. PC: platelet concentrations. ES: average value for implant survival rate. CI: confidential interval.

upregulating osteoblast proliferation and regeneration of periodontal ligament cells ${ }^{59}$. Furthermore, the slow platelets aggregation allows for more cytokines to be embedded within such mesh-like structures, and their slow degradation rates allows for sustained release of growth factors into the surrounding tissue ${ }^{12}$.

Another distinguished feature of platelet concentrations is their softness and flexibility, which can act like a buffer material between protruded bone fragments/implant screws and Schneiderian membrane ${ }^{9,53-55}$. Compared with the traditional bone grafts that contain particles with sharp edges, the liquid/gel-like substrates of platelet concentrations are protective to the delicate Schneiderian membrane from being penetrated ${ }^{9,53-55}$.

In this study, initially we analyzed the implant survival rate (ISR) within non-grafted TSFE studies among different $\mathrm{RBH}$, and no significant differences were observed on 1-year ISR among groups with different average $\mathrm{RBH}$ within non-grafted TSFE group, even cases with limited $\mathrm{RBH}(<4 \mathrm{~mm})$ obtained favorable 1-year ISR ${ }^{51,60,61}$. This confirmed that limited RBH does not impact the implant survival for non-grafted TSFE surgeries, as long as secured primary stabilities are obtained on implant $s^{51,60,61}$. Furthermore, the increase in bone-implant contact area and the compression of bone during TSFE augments the bone density around implant sites. This in turn can enhance the primary stability of implant at TSFE sites, which effectively reduces the fretting of implants and promotes osseointegration at implant-bone interface ${ }^{51}$.

To investigate the enhancement of osseointegration and bone stability by adding PCs in TSFE sites, we performed meta-analysis on average 1-year ISR between non-grafted TSFE and PC-TSFE, which has not been reported before. Result showed no significant differences on 1-year ISR between the two groups, indicating that adding PCs in TSFE sites will not significantly enhance the strength and stability of osseointegration around implants. It is noteworthy that TSFE will press implant screws and adjacent bone into maxillary sinus, which are capable of supporting the apically displaced Schneiderian membrane, thus maintaining the stable osteogenic chamber ${ }^{62}$.

Furthermore, in the studies with MBL, there was no significant difference between the non-grafted TSFE and PC-TSFE groups. This can be attributed to the fact that PCs were lifted to the apical region of surgical sites, not the crestal region which could not directly influence the MBL during the constant masticatory cycles. It is noteworthy that MBL around implants is influenced by various factors including smoking, platform-switching technique at implant-abutment interfere, surface characteristics of implants/abutments, surgical trauma and functional loadings ${ }^{63-65}$. In the current study, the average 1 -year MBL of non-grafted TSFE $(0.73 \mathrm{~mm}$, with $95 \% \mathrm{CI}=0.33-$ $1.13 \mathrm{~mm}$ ) was shown to be acceptable for the clinical application. Enhancement of peri-implant bone density alleviates the MBL, and the TSFE increases the density of peri-implant cancellous bone; hence the non-grafted TSFE can supports primary stability and peri-implant bone density (towards long term implant success) ${ }^{66-68}$.

For the ESBG data, although the PC-TSFE group showed a slightly higher 1-year average ESBG, there was no significant difference with non-grafted TSFE group. This is because PCs are flexible and gel-like substrates, 


\begin{tabular}{|c|c|c|c|}
\hline Study & & & $\%$ \\
\hline ID & & $\mathrm{ES}(95 \% \mathrm{CI})$ & Weight \\
\hline $\mathrm{RBH}<4 \mathrm{~mm}$ & i & & \\
\hline Nedir (2017) & $\rightarrow$ & $0.99(0.93,1.04)$ & 9.02 \\
\hline Gu Y X (2016) & $\rightarrow$ & $0.93(0.86,1.01)$ & 4.47 \\
\hline Subtotal $(\mathrm{I}-$ squared $=5.6 \%, \mathrm{p}=0.303)$ & $\diamond$ & $0.97(0.92,1.02)$ & 13.49 \\
\hline . & 1 & & \\
\hline $\mathrm{RBH}=4-6 \mathrm{~mm}$ & $i$ & & \\
\hline Nedir (2016) & $\leftarrow$ & $0.99(0.95,1.03)$ & 19.15 \\
\hline Si M S (2013) & $\rightarrow$ & $0.99(0.94,1.04)$ & 12.38 \\
\hline Caban J (2017) & $\rightarrow-1$ & $0.90(0.80,1.00)$ & 2.79 \\
\hline Subtotal $(\mathrm{I}-\mathrm{squared}=27.5 \%, \mathrm{p}=0.252)$ & 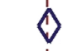 & $0.98(0.94,1.02)$ & 34.32 \\
\hline . & $\begin{array}{c}1 \\
1 \\
1\end{array}$ & & \\
\hline $\mathrm{RBH}>6 \mathrm{~mm}$ & 1 & & \\
\hline Si M S (2016) & $\rightarrow$ & $0.96(0.93,1.00)$ & 20.43 \\
\hline D.Spinelli (2015) & $\leftarrow$ & $0.98(0.94,1.01)$ & 22.84 \\
\hline Robert F (2012) & $\rightarrow$ & $0.95(0.90,1.01)$ & 8.92 \\
\hline Subtotal $(I-$ squared $=0.0 \%, p=0.742)$ & $\phi$ & $0.97(0.95,0.99)$ & 52.19 \\
\hline . & i & & \\
\hline Overall $(\mathrm{I}-$ squared $=0.0 \%, \mathrm{p}=0.663)$ & 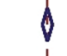 & $0.97(0.96,0.99)$ & 100.00 \\
\hline NOTE: Weights are from random effects analysis & 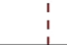 & & \\
\hline $\begin{array}{c}1 \\
-1.04\end{array}$ & 1. & & \\
\hline
\end{tabular}

Figure 3. The meta-analysis on 1-year implant survival rate among subgroups with varied RBH within nongrafted TSFE. Forest plot was generated by Stata 14.0, randomized model was applied for the meta-analysis. RBH: residual bone height; TSFE: transcrestal sinus floor elevation. ES: average value for implant survival rate; CI: confidential interval.

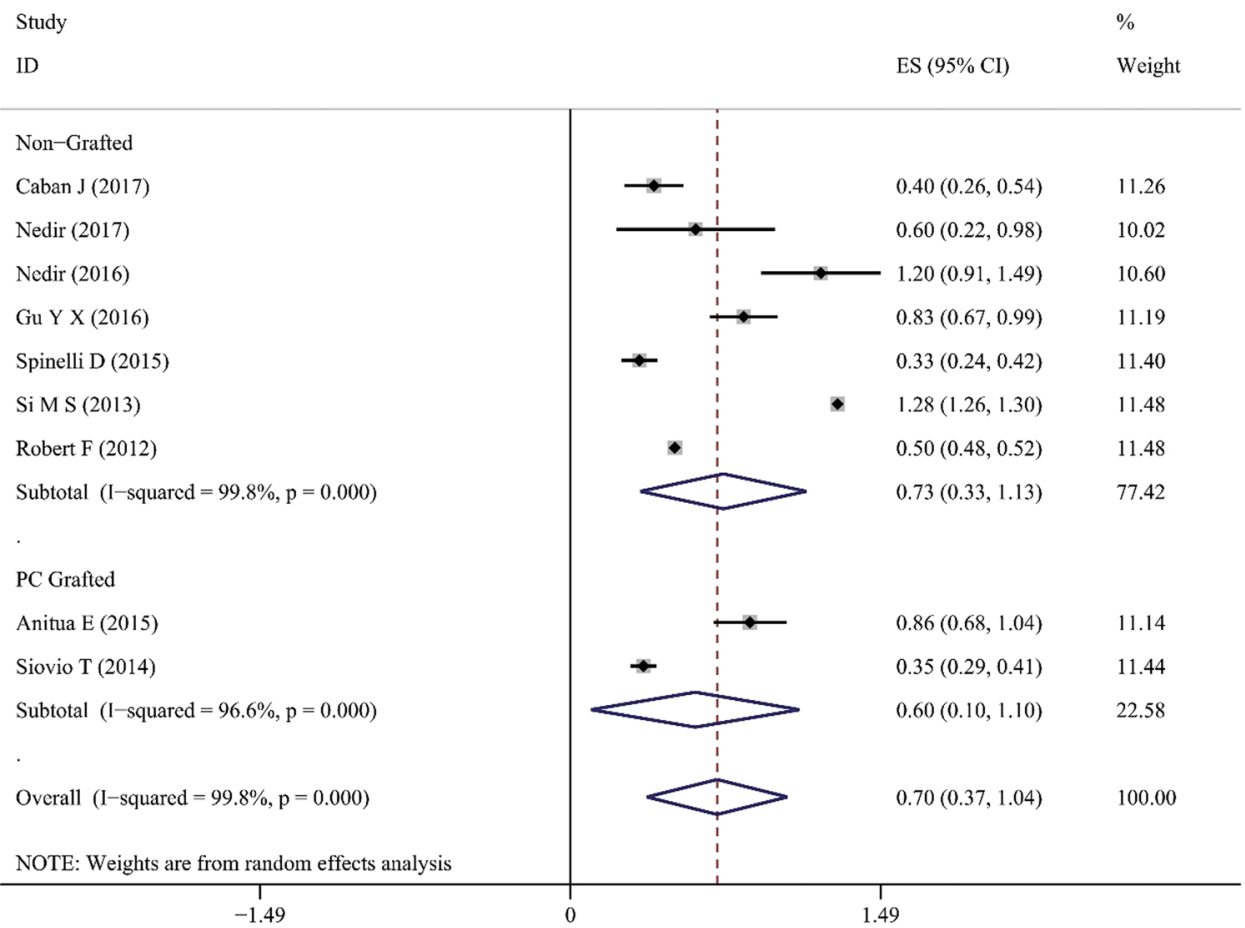

Figure 4. Result of meta-analysis on 1-year marginal bone loss (MBL) for non-grafted and PC-TSFE groups. Forest plot was generated by Stata 14.0, randomized model was applied for the meta-analysis. PC: platelet concentrations. ES: average value for 1-year MBL; CI: confidential interval. 


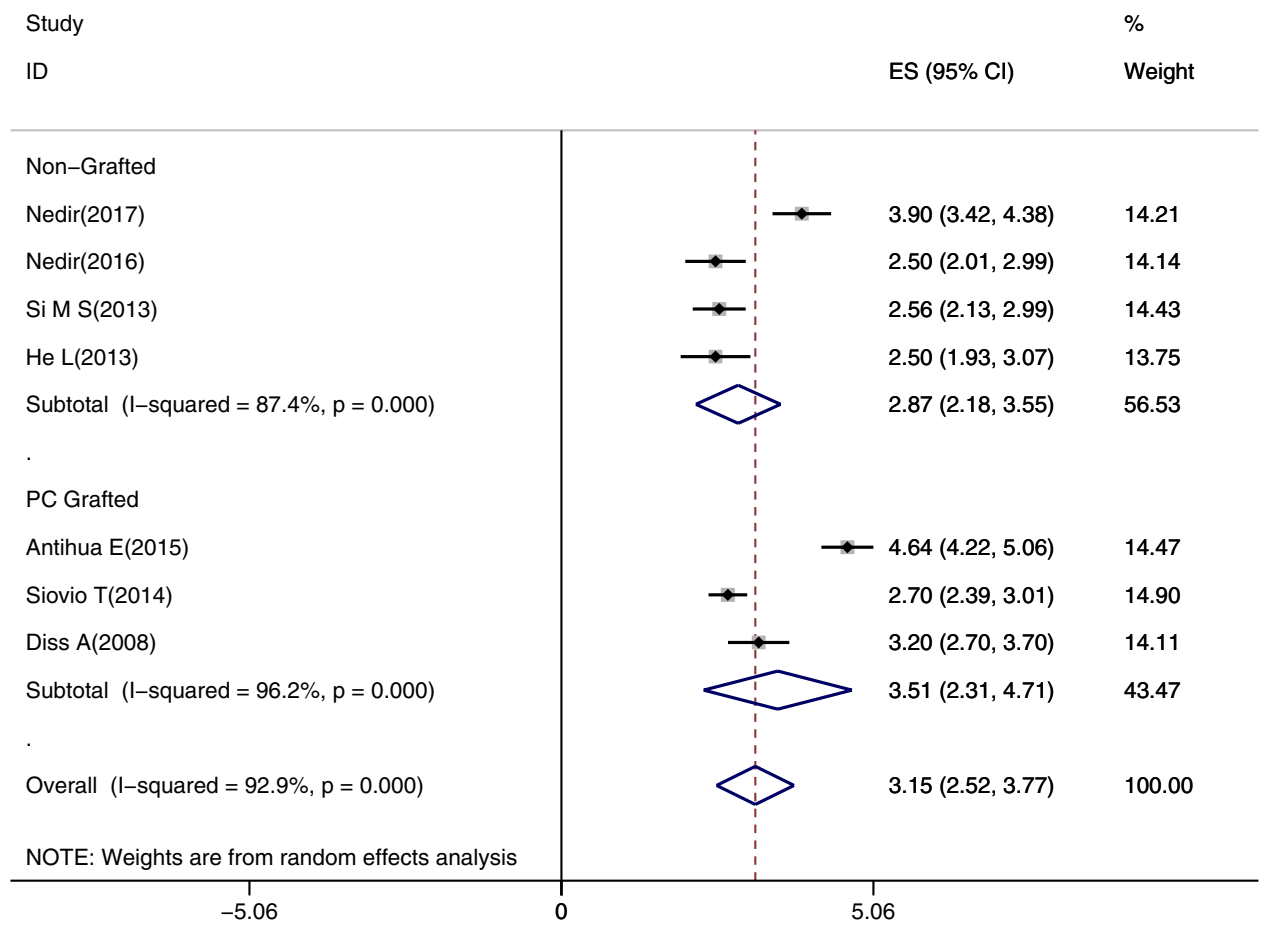

Figure 5. Result of meta-analysis on 1-year endo-sinus bone gain (ESBG) between non-grafted and PC-TSFE groups. Forest plot was generated by Stata 14.0, randomized model was applied for the meta-analysis. PC: platelet concentrations. ES: average value for 1-year ESBG; CI: confidential interval.

which are not mechanically rigid to maintain extra augmentations of Schnederian membrane in TSFE sites. Furthermore, although PCs are are known to stimulate proliferation, migration and differentiation of adjacent osteoblasts/fibroblasts, their biodegradation characteristics does not continuously support the elevated Schneiderian membrane ${ }^{69-71}$. It is noteworthy that the chamber for bone regeneration at TSFE sites is maintained by the pressed implants and the displaced maxillary bone, thus the non-grafted TSFE is sufficient towards maintaining the vertical bone gain. These results correlates with the study by Liu et al., which reported that applying PCs as adjunctive to bone substitutions into MSFA sites did not show significant enhancement of regeneration and formation of new bone around implants ${ }^{72}$. Moreover, PCs will resorb within a few days after being implanted in human body. Such rapid degradation prevents them from constantly releasing cytokines during the bone regeneration phase in surgical sites ${ }^{72}$.

In summary, PCs contain various cytokines to promote bone regeneration. However, they are unable to significantly enhance the stability and vertical quantity of newly regenerated bone around the implant surfaces in TSFE sites. This is attributed to the inappropriate mechanical (softness and flexibility) and biodegradation characteristics of platelet concentrations, which prevents them from maintaining an osteogenic chamber by continuously supporting the Schneiderian membrane. Moreover, non-grafted TSFE is a reliable treatment modality for sites needing MSFA, even with limited RBH $(<4 \mathrm{~mm})$. To optimize their osteogenic potential, combining PCs with mechanically rigid and slow degrading bone substitutions to continuously support osteogenic chamber, can promote bone quantity and quality in TSFE sites. However, this represents a research gap and hence needs further investigations. Additionally, for further enhancement of PC-induced bone regeneration, more histological studies on bone formation pattern and process (including vascularizationand mineralization) are needed. Finally, the clinical studies on PC-TSFE are limited and most of them are based on short-term post-surgical data (mainly 1 year), which is a shortcoming for the current systematic review and requires long-term investigations for further advancements.

\section{Conclusion}

This systematic review and meta-analysis compared the therapeutic outcomes of non-grafted transcrestal sinus floor elevation (TSFE) with platelet concentrations grafted TSFE (PC-TSFE). The results indicated no significant enhancements on 1-year implant survival rates, marginal bone loss (MBL) and endosinus bone gain (ESBG) of PC-TSFE group as compared to non-grafted TSFE group. Moreover, among subgroups of different residual bone height $(\mathrm{RBH})$ within the non-grafted TSFE group, no significant differences were shown on their implant survival rates. The results confirm that adding platelet concentrations into maxillary sinus could not provide significant enhancements to the therapeutic outcomes of TSFE. However, to provide strong evidence on the therapeutic outcomes non-grafted and platelet grafted TSFE long term clinical studies must be conducted. Finally, to utilize the osteogenic potential of platelet concentrations and obtain an optimized bone quality/quantity in TSFE sites, further clinical and histological studies are needed, specially with the combined graft of platelet concentrations with 
other bone substitutes into TSFE sites. To date, the non-grafted TSFE remains a reliable therapeutic option for surgical sites that need maxillary sinus floor elevation (MSFA), even in cases with limited RBH.

\section{Methods and materials}

Search strategies. The PICO elements for this study are P (Problem): insufficient bone quantity at maxillary molar sites; I (intervention/indicator): grafting platelet concentrations in TSFE sites; C (comparison): non-grafted TSFE surgeries; $\mathrm{O}$ (outcome of interest): enhanced bone regeneration and osseointegration. The initial literature search was conducted in the Scopus, Pubmed and Cochrane library database. All relevant studies until April 2019 were included relating to indirect/transcrestal maxillary sinus elevation (TSFE). A search strategy of Mesh term or Keywords included a combination of ["maxillary sinus floor augmentation (MSFA)" OR "maxillary sinus lift" OR "maxillary sinus augmentation" OR "maxillary sinus elevation"] and ["transcrestal" OR "osteotomy" OR "transcrestal sinus floor elevation (TSFE)" OR "indirect sinus floor elevation" OR "osteotomy sinus floor elevation (OSFE)"] (processed by TG and ZF). After removing the duplications, a sequential selection of title, abstract and full text reading were performed on the residual literatures and finally selected. These articles included the studies relating to non-grafted TSFE and PC-grafted TSFE. Search and selection of literature was conducted by two reviewers (TG and ZF). If disagreements occurred between these two reviewers, a third reviewer (ZS) was recommended to judge the disagreement. All the literature were managed by Endnote X9.

Inclusion criteria. Randomized controlled trials (RCTs), controlled clinical trials and observational studies (prospective and retrospective cohort studies) on the studies related to non-grafted TSFE or PC-grafted TSFE were included in this study. Minimum implants enrolled in each study was 15, and the shortest post-surgical observation period was 6 months. Moreover, demographic data of implants and patients (brand of implants, surgical methods, implant and patient numbers) must be clearly described. For studies that compare the therapeutic outcomes between non-grafted TSFE and bone-substitutions grafted TSFE, the outcomes of the non-grafted groups were extracted and analyzed in this paper. For studies which compared PC-grafted TSFE with bone substitutions grafted TSFE, data of the PC-TSFE group were extracted and compared.

Exclusion criteria. Case reports, technical reports, conference articles, animal experiments, in vitro studies, finite element analysis, reviews and letters to the editors were all excluded. Clinical studies with insufficient post-surgical follow-up visiting periods, or inaccurately described therapeutic outcomes, and those unable to clearly define demographics were also excluded.

Study selection and data extraction. Study selections were conducted by two researchers (TG and ZF), and the detailed process is presented in Fig. 1. When disagreements occurred, a third person (ZS) was asked to review and decide. Data of each selected study was extracted by two reviewers (TG and ZF), including: (1) demographics: authors, year of publishing, implant systems, numbers of implants and patients; (2) individual information: surgical methods and residual bone height $(\mathrm{RBH})$; and (3) therapeutic outcomes: implant survival rate (ISR), endo-sinus bone gain (ESBG) and crestal/marginal bone loss (CBL/MBL). For studies that reported the therapeutic outcomes in sequential follow-up visit time, the data at all follow-up visit time points were extracted into the systematic review.

Literature quality assessment. Quality assessment on the selected literature was performed by two researchers (TG and FZ). As showed in Table 1, these controlled studies were assessed by eight criteria under the Cochrane Collaboration guidelines ${ }^{73}$. An assessed study was confined as "low risk of bias" if all the criteria were answered "Yes". If 1-2 clauses were "No", the risk bias was defined as "moderate". If more than 2 clauses were "No", the risk of bias was outlined as "high". Newcastle-Ottawa Scale (NOS) was applied for assessing the quality of non-randomized studies (prospective and retrospective cohort studies), just as Table 2 describes, with the maximum possible score 9 . Any study scored 7 or more was determined as high quality with low risk of bias ${ }^{73}$.

Data synthesis and meta-analysis. Stata MP 14.1 software was applied to process the meta-analysis, randomized model was applied to process the meta-analysis, with heterogeneity indicated by I-square values. To include studies having a $100 \%$ implant survival rate, we modified the rates with the formula: [modified survival rate $=($ number of survived implants -0.25$) /$ (number of total implants) $* 100 \%]$ in Stata MP 14.1. Considering the data of ISR, MBL and ESBG were based on the 1-year follow-up in majority of the literature, the 1-year post-surgical data of these parameters were considered in the meta-analysis. Moreover, to investigate the clinical reliability of non-grafted TSFE in cases with limited RBH, study on 1-year ISR of non-grafted TSFE was performed among its subgroups with varied $\mathrm{RBH}$ values.

Variables processing and comparison. Since the 1-year ISR values pooled by Stata 14.0 were only accurate to their single digital units (eg. $0.99=99 \%$ ), thus observation and descriptions were applied on these pooled values. For exploring the differences of 1-year MBL and ESBG between non-grafted and PC-grafted TSFE, these data were pooled and compared as described below:

Stata 14.0 applies $95 \%$ confidence interval (CI) to display the distribution of continuous variables, as indicated by Cochrane Handbook for Systematic Reviews (Version 5.0.2 Chapter 7.7.3.2.), to calculate the standard deviation (SD) of each group from the $95 \% \mathrm{CI}$, the equation below was applied ${ }^{74}$ :

$$
S D=\sqrt{n} \times(\text { upper limit }- \text { lower limit }) / 3.92
$$


As grafting PC is the intervention (I) in this study, thus the changes of average value, SD and standard error (SE) of each variable after intervention were calculated as the equations below, indicated by Cochrane Handbook for Systematic Reviews (Version 5.0.2 Chapter 16.1.3.2. ${ }^{74}$ :

$$
\begin{gathered}
\text { Mean }(E, \text { change })=\text { Mean }(P C)-\text { Mean }(\text { Non }) \\
S D(E, \text { change })=\sqrt{S D(P C)^{2}+S D(\text { Non })^{2}-2 \times \text { Corr } \times S D(P C) \times S D(\text { Non })} \\
S E(E, \text { change })=\frac{S D(E, \text { change })}{\sqrt{n}}
\end{gathered}
$$

In the equations above, Non represents non-grafted TSFE group and $P C$ represents PC-grafted TSFE group, and Corr value was determined as 0.40 , if the $95 \% \mathrm{CI}\left(\mathrm{Mean}_{(\mathrm{E} \text {, change })} \pm 1.96 \times \mathrm{SE}_{(\mathrm{E} \text {, change })}\right)$ of changes in any variables contain 0 , it is indicated that such variable had no significant enhancements/differences after grafted by PC, which is the intervention (I).

Received: 14 October 2019; Accepted: 11 March 2020;

Published online: 03 April 2020

\section{References}

1. Chanavaz, M. Maxillary sinus: anatomy, physiology, surgery, and bone grafting related to implantology-eleven years of surgical experience (1979-1990). J. Oral. Implantol. 16(3), 199-209 (1990).

2. Woo, I. \& Le, B. T. Maxillary sinus floor elevation: review of anatomy and two techniques. Implant. Dent. 13(1), 28-32 (2004).

3. Boyne, P. J. \& James, R. A. Grafting of the maxillary sinus floor with autogenous marrow and bone. J. Oral. Surg. 38(8), 613-6 (1980).

4. Trombelli, L. et al. Minimally invasive transcrestal sinus floor elevation with graft biomaterials. A randomized clinical trial. Clin. Oral. Implant. Res. 23(4), 424-32 (2012).

5. Emmerich, D., Att, W. \& Stappert, C. Sinus floor elevation using osteotomes: a systematic review and meta-analysis. J. Periodontol. 76(8), 1237-51 (2005).

6. Bassi, A. P. et al. Maxillary sinus lift without grafting, and simultaneous implant placement: a prospective clinical study with a 51-month follow-up. Int. J. Oral. Maxillofac. Surg. 44(7), 902-7 (2015).

7. Taschieri, S. \& Del Fabbro, M. Postextraction osteotome sinus floor elevation technique using plasma-rich growth factors. Implant. Dent. 20(6), 418-24 (2011).

8. Moraschini, V. et al. Maxillary sinus floor elevation with simultaneous implant placement without grafting materials: a systematic review and meta-analysis. Int. J. Oral. Maxillofac. Surg. 46(5), 636-647 (2017).

9. Khorshidi, H. et al. Comparison of the Mechanical Properties of Early Leukocyte- and Platelet-Rich Fibrin versus PRGF/Endoret Membranes. Int. J. Dent. 2016, 1849207-1849207 (2016).

10. Sani, E. et al. Sinus membrane elevation in combination with placement of blasted implants: A 3-year case report of sinus augmentation without grafting material. Int. J. Oral. Maxillofac. Surg. 37(10), 966-969 (2008).

11. Bruschi, G. B. et al. Transcrestal sinus floor elevation: a retrospective study of 46 patients up to 16 years. Clin. Implant. Dent. Relat. Res. 14(5), 759-67 (2012).

12. He, L. et al. A comparative study of platelet-rich fibrin (PRF) and platelet-rich plasma (PRP) on the effect of proliferation and differentiation of rat osteoblasts in vitro. Oral. Surg. Oral Med. Oral Pathol. Oral Radiol. Endod. 108(5), 707-13 (2009).

13. Dohan, D. M. et al. Platelet-rich fibrin (PRF): a second-generation platelet concentrate. Part III: leucocyte activation: a new feature for platelet concentrates? Oral. Surg. Oral Med. Oral Pathol. Oral Radiol. Endod. 101(3), e51-5 (2006).

14. Roffi, A. et al. Does PRP enhance bone integration with grafts, graft substitutes, or implants? A systematic review. BMC Musculoskelet. Disord. 14, 330 (2013).

15. Paknejad, M. et al. Evaluation of the Effect of Plasma Rich in Growth Factors (PRGF) on Bone Regeneration. J. Dent. 9(1), 59-67 (2012).

16. Liao, H. T., Marra, K. G. \& Rubin, J. P. Application of platelet-rich plasma and platelet-rich fibrin in fat grafting: basic science and literature review. Tissue Eng. Part. B Rev. 20(4), 267-76 (2014).

17. Fernandez-Moure, J. S. et al. Platelet-rich plasma: a biomimetic approach to enhancement of surgical wound healing. J. Surg. Res. 207, 33-44 (2017).

18. Anitua, E. et al. Association of transalveolar sinus floor elevation, platelet rich plasma, and short implants for the treatment of atrophied posterior maxilla. Clin. Oral. Implant. Res. 26(1), 69-76 (2015).

19. Taschieri, S., Corbella, S. \& Del Fabbro, M. Mini-invasive osteotome sinus floor elevation in partially edentulous atrophic maxilla using reduced length dental implants: interim results of a prospective study. Clin. Implant. Dent. Relat. Res. 16(2), 185-93 (2014).

20. Kim, J. M. et al. Flapless transcrestal sinus augmentation using hydrodynamic piezoelectric internal sinus elevation with autologous concentrated growth factors alone. Implant. Dent. 23(2), 168-74 (2014).

21. Diss, A. et al. Osteotome sinus floor elevation using Choukroun's platelet-rich fibrin as grafting material: a 1-year prospective pilot study with microthreaded implants. Oral. Surg. Oral Med. Oral Pathol. Oral Radiol. Endod. 105(5), 572-9 (2008).

22. Nedir, R. et al. Osteotome sinus floor elevation technique without grafting material: 3-year results of a prospective pilot study. Clin. Oral. Implant. Res. 20(7), 701-7 (2009).

23. Nedir, R. et al. Osteotome sinus floor elevation technique without grafting: a 5-year prospective study. J. Clin. Periodontol. 37(11), 1023-8 (2010).

24. Nedir, R. et al. Osteotome sinus floor elevation with and without grafting material in the severely atrophic maxilla. A 1 -year prospective randomized controlled study. Clin. Oral. Implant. Res. 24(11), 1257-64 (2013).

25. Nedir, R. et al. Short Implants Placed with or without Grafting in Atrophic Sinuses: The 3-Year Results of a Prospective Randomized Controlled Study. Clin. Implant. Dent. Relat. Res. 18(1), 10-8 (2016).

26. Fermergard, R. \& Astrand, P. Osteotome sinus floor elevation and simultaneous placement of implants-a 1-year retrospective study with Astra Tech implants. Clin. Implant. Dent. Relat. Res. 10(1), 62-9 (2008).

27. Anitua, E., Flores, J. \& Alkhraisat, M. H. Transcrestal Sinus Lift Using Platelet Concentrates in Association to Short Implant Placement: A Retrospective Study of Augmented Bone Height Remodeling. Clin. Implant. Dent. Relat. Res. 18(5), 993-1002 (2016).

28. Toffler, M., Toscano, N. \& Holtzclaw, D. Osteotome-mediated sinus floor elevation using only platelet-rich fibrin: an early report on 110 patients. Implant. Dent. 19(5), 447-56 (2010).

29. Gabbert, O. et al. Implants placed in combination with an internal sinus lift without graft material: an analysis of short-term failure. J. Clin. Periodontol. 36(2), 177-83 (2009). 
30. Markovic, A. et al. Two-Center Prospective, Randomized, Clinical, and Radiographic Study Comparing Osteotome Sinus Floor Elevation with or without Bone Graft and Simultaneous Implant Placement. Clin. Implant. Dent. Relat. Res. 18(5), 873-882 (2016).

31. Agamy, E. M. \& Niedermeier, W. Indirect sinus floor elevation for osseointegrated prostheses. A 10-year prospective study. J. Oral. Implantol. 36(2), 113-21 (2010).

32. French, D. et al. Trifactorial classification system for osteotome sinus floor elevation based on an observational retrospective analysis of 926 implants followed up to 10 years. Quintessence Int. 46(6), 523-30 (2015).

33. Chen, Y. et al. Inlay osteotome sinus floor elevation with concentrated growth factor application and simultaneous short implant placement in severely atrophic maxilla. Sci. Rep. 6, 27348 (2016).

34. Franceschetti, G. et al. Learning Curve of a Minimally Invasive Technique for Transcrestal Sinus Floor Elevation: A Split-Group Analysis in a Prospective Case Series With Multiple Clinicians. Implant. Dent. 24(5), 517-26 (2015).

35. Caban, J., Fermergard, R. \& Abtahi, J. Long-term evaluation of osteotome sinus floor elevation and simultaneous placement of implants without bone grafts: 10-Year radiographic and clinical follow-up. Clin. Implant. Dent. Relat. Res. 19(6), 1023-1033 (2017).

36. Nedir, R. et al. Short implants placed with or without grafting into atrophic sinuses: the 5-year results of a prospective randomized controlled study. Clin. Oral. Implant. Res. 28(7), 877-886 (2017).

37. Nedir, R. et al. Osteotome Sinus Floor Elevation without Grafting: A 10-Year Prospective Study. Clin. Implant. Dent. Relat. Res. 18(3), 609-17 (2016).

38. Si, M. S. et al. Long-term outcomes of osteotome sinus floor elevation without bone grafts: a clinical retrospective study of 4-9 years. Clin. Oral. Implant. Res. 27(11), 1392-1400 (2016).

39. Gu, Y. X. et al. Transalveolar sinus floor elevation using osteotomes without grafting in severely atrophic maxilla: a 5-year prospective study. Clin. Oral. Implant. Res. 27(1), 120-5 (2016).

40. Spinelli, D. et al. Transcrestal guided sinus lift without grafting materials: a 36 months clinical prospective study. Oral. Implantol. 8(2-3), 74-86 (2015).

41. Brizuela, A. et al. Osteotome sinus floor elevation without grafting material: Results of a 2-year prospective study. J. Clin. Exp. Dent. 6(5), e479-84 (2014).

42. Si, M. S. et al. Osteotome sinus floor elevation with or without grafting: a 3-year randomized controlled clinical trial. J. Clin. Periodontol. 40(4), 396-403 (2013).

43. Volpe, S. et al. Clinical outcomes of an osteotome technique and simultaneous placement of Neoss implants in the posterior maxilla. Clin. Implant. Dent. Relat. Res. 15(1), 22-8 (2013).

44. He, L., Chang, X. \& Liu, Y. Sinus floor elevation using osteotome technique without grafting materials: a 2-year retrospective study. Clin. Oral. Implant. Res. 24(Suppl A100), 63-7 (2013).

45. Fermergard, R. \& Astrand, P. Osteotome sinus floor elevation without bone grafts-a 3-year retrospective study with Astra Tech implants. Clin. Implant. Dent. Relat. Res. 14(2), 198-205 (2012).

46. Lai, H. C. et al. Osteotome sinus floor elevation with or without grafting: a preliminary clinical trial. Clin. Oral. Implant. Res. 21(5), $520-6(2010)$

47. Schleier, P. et al. Simultaneous dental implant placement and endoscope-guided internal sinus floor elevation: 2-year post-loading outcomes. Clin. Oral. Implant. Res. 19(11), 1163-70 (2008).

48. Srouji, S. et al. The Schneiderian membrane contains osteoprogenitor cells: in vivo and in vitro study. Calcif. Tissue Int. 84(2), 138-45 (2009).

49. Gruber, R. et al. Porcine sinus mucosa holds cells that respond to bone morphogenetic protein (BMP)-6 and BMP-7 with increased osteogenic differentiation in vitro. Clin. Oral. Implant. Res. 15(5), 575-80 (2004).

50. Duan, D. H. et al. Graft-Free Maxillary Sinus Floor Elevation: A Systematic Review and Meta-Analysis. J. Periodontol. 88(6), 550-564 (2017)

51. Pérez-Martínez, S. et al. Indirect sinus lift without bone graft material: Systematic review and meta-analysis. J. Clin. Exp. Dent. 7(2), e316-e319 (2015)

52. Silva, L. D. et al. Maxillary sinus lift surgery-with or without graft material? A systematic review. Int. J. Oral. Maxillofac. Surg. 45(12), 1570-1576 (2016).

53. Castro, A. B. et al. Regenerative potential of leucocyte- and platelet-rich fibrin. Part B: sinus floor elevation, alveolar ridge preservation and implant therapy. A systematic review. J. Clin. Periodontol. 44(2), 225-234 (2017).

54. Aoki, N. et al. Sinus Augmentation by Platelet-Rich Fibrin Alone: A Report of Two Cases with Histological Examinations. Case Rep. Dent. 2016, 7 (2016).

55. Kumar, N. K. et al. Comparative study of alveolar bone height and implant survival rate between autogenous bone mixed with platelet rich plasma versus venous blood for maxillary sinus lift augmentation procedure. J. Maxillofac. oral. Surg. 14(2), 417-422 (2015).

56. Rodriguez, I. A. et al. Platelet-rich plasma in bone regeneration: engineering the delivery for improved clinical efficacy. Biomed. Res. Int. 2014, 392398 (2014).

57. Dohan, D. M. et al. Platelet-rich fibrin (PRF): a second-generation platelet concentrate. Part II: platelet-related biologic features. Oral. Surg. Oral Med. Oral Pathol. Oral Radiol. Endod. 101(3), e45-50 (2006).

58. Chen, X. et al. Effect of Concentrated Growth Factor (CGF) on the Promotion of Osteogenesis in Bone Marrow Stromal Cells (BMSC) in vivo. Sci. Rep. 8(1), 5876 (2018).

59. Qin, J. et al. Concentrated growth factor promotes Schwann cell migration partly through the integrin beta1-mediated activation of the focal adhesion kinase pathway. Int. J. Mol. Med. 37(5), 1363-70 (2016).

60. Riben, C. \& Thor, A. The Maxillary Sinus Membrane Elevation Procedure: Augmentation of Bone around Dental Implants without Grafts\&\#8212;A Review of a Surgical Technique. Int. J. Dent. 2012, 9 (2012).

61. Hegde, R., Prasad, K. \& Shroff, K. K. Maxillary sinus augmentation using sinus membrane elevation without grafts - A Systematic Review. J. Indian. Prosthodont. Soc. 16(4), 317-322 (2016).

62. Stefanski, S., Svensson, B. \& Thor, A. Implant survival following sinus membrane elevation without grafting and immediate implant installation with a one-stage technique: an up-to-40-month evaluation. 28(11), 1354-1359 (2017).

63. Starch-Jensen, T. et al. Maxillary Sinus Floor Augmentation With Synthetic Bone Substitutes Compared With Other Grafting Materials: A Systematic Review and Meta-analysis. Implant. Dent. 27(3), 363-374 (2018).

64. Shanbhag, S., Shanbhag, V. \& Stavropoulos, A. Volume changes of maxillary sinus augmentations over time: a systematic review. Int. J. Oral. Maxillofac. Implant. 29(4), 881-92 (2014).

65. Sanchez-Siles, M. et al. Incidence of peri-implantitis and oral quality of life in patients rehabilitated with implants with different neck designs: A 10-year retrospective study. J. Craniomaxillofac Surg. 43(10), 2168-74 (2015).

66. Cowin, S. C. Bone stress adaptation models. J. Biomech. Eng. 115(4b), 528-33 (1993).

67. van Steenberghe, D. A retrospective multicenter evaluation of the survival rate of osseointegrated fixtures supporting fixed partial prostheses in the treatment of partial edentulism. J. Prosthet. Dent. 61(2), 217-23. (1989).

68. Khalifa, A. K. et al. To what extent residual alveolar ridge can be preserved by implant? A systematic review. Int. J. Implant. Dent. 2(1), 22-22 (2016).

69. Anitua, E. et al. PRGF exerts more potent proliferative and anti-inflammatory effects than autologous serum on a cell culture inflammatory model. Exp. Eye Res. 151, 115-21 (2016). 
70. Su, C. Y. et al. In vitro release of growth factors from platelet-rich fibrin (PRF): a proposal to optimize the clinical applications of PRF. Oral. Surg. Oral Med. Oral Pathol. Oral Radiol. Endod. 108(1), 56-61 (2009).

71. Choukroun, J. et al. Platelet-rich fibrin (PRF): a second-generation platelet concentrate. Part V: histologic evaluations of PRF effects on bone allograft maturation in sinus lift. Oral. Surg. Oral Med. Oral Pathol. Oral Radiol. Endod. 101(3), 299-303 (2006).

72. Liu, R. et al. Effectiveness of Platelet-Rich Fibrin as an Adjunctive Material to Bone Graft in Maxillary Sinus Augmentation: A MetaAnalysis of Randomized Controlled Trails. BioMed. Res. Int. 2019, 7267062-7267062 (2019).

73. Jackson, N. \& Waters, E. Criteria for the systematic review of health promotion and public health interventions. Health Promot. Int. 20(4), 367-74 (2005)

74. Higgins, J. P. T. et al. Cochrane Handbook for Systematic Reviews of Interventions version 6.0. (2019: Cochrane).

\section{Acknowledgements}

Tianqi Guo is supported by the UQ Graduate School Scholarships (UQGSS) funded by the University of Queensland, Australia. Zhen Fan is supported by the National Natural Science Foundation Youth Fund Project. Grant Number: 81400485. Karan Gulati is supported by the National Health and Medical Research Council (NHMRC) Early Career Fellowship (ECF), Peter Doherty-Australian Biomedical Fellowship (APP1140699).

\section{Author contributions}

Tianqi Guo: Study design, data collection and analysis, drafting paper. Karan Gulati: Study design, critical revisions, data collection and analysis. Ziyun Shen: Study design, drafting paper. Pingping Han: Critical revisions, statistics. Zhen Fan: Study design, critical revisions, approval of article, funding secured.

\section{Competing interests}

The authors declare no competing interests.

\section{Additional information}

Correspondence and requests for materials should be addressed to Z.F.

Reprints and permissions information is available at www.nature.com/reprints.

Publisher's note Springer Nature remains neutral with regard to jurisdictional claims in published maps and institutional affiliations.

(c) (i) Open Access This article is licensed under a Creative Commons Attribution 4.0 International License, which permits use, sharing, adaptation, distribution and reproduction in any medium or format, as long as you give appropriate credit to the original author(s) and the source, provide a link to the Creative Commons license, and indicate if changes were made. The images or other third party material in this article are included in the article's Creative Commons license, unless indicated otherwise in a credit line to the material. If material is not included in the article's Creative Commons license and your intended use is not permitted by statutory regulation or exceeds the permitted use, you will need to obtain permission directly from the copyright holder. To view a copy of this license, visit http://creativecommons.org/licenses/by/4.0/.

(c) The Author(s) 2020 